www.nature.com/pj

\title{
Mussel-mimetic strong adhesive resin from bio-base polycoumarates
}

\author{
Daisaku Kaneko $^{1}$, Siqing Wang ${ }^{1}$, Keitaro Matsumoto ${ }^{1}$, Shougo Kinugawa ${ }^{1}$, Katsuaki Yasaki ${ }^{1}$, Dam Hieu Chi ${ }^{2}$ \\ and Tatsuo Kaneko ${ }^{1}$
}

We have synthesized novel Mussel-mimetic adhesive resin from biomass monomers. Because the adhesive resin contains catechol groups at its chain ends, which is similar to the chemical structure of 3,4-dihydroxyphenyl-L-alanine, produced by Mussel, it demonstrates strong adhesive characteristics onto organic and/or inorganic surfaces beyond those of the conventional instant superglue. The origin of this strong adhesive characteristic was attributed to strong hydrogen bond interaction between our new resins and oxidized and/or $\mathrm{OH}$ groups on the substrates.

Polymer Journal (2011) 43, 855-858; doi:10.1038/pj.2011.77; published online 17 August 2011

Keywords: adhesive; hyper-branched polymer; mussel-mimetic; polycoumarates

\section{INTRODUCTION}

It is well known that Mussels can adhere strongly to rocks (on organic or inorganic surfaces) by producing 3,4-dihydroxyphenyl-L-alanine, which contains the catechol group. ${ }^{1-5}$ The 3,4-dihydroxyphenyl-Lalanine-containing catechol group can adhere strongly and reversibly to both organic and inorganic surfaces. Although many attempts have been made to clarify the adhesion mechanism, ${ }^{6,7}$ the details are not completely understood. However, potential applications of this adhesive function have fascinated many researchers. For example, the group of Messersmith and Miller ${ }^{8-12}$ has demonstrated to mimic a material that mimics the behavior of mussel adhesive in the form of adhesive sheets. Although this strong adhesion has been attributed to hydrogen bond interactions or covalent bonding between the catechol groups, the adhesive mechanism is not completely understood.

We have previously reported the polymerization of the catecholderivative monomer caffeic acid (3,4-dihydroxycinnamic acid, DHCA, Figure 1a) to prepare wholly aromatic polyarylates. Their copolymerization DHCA with p-coumaric acid (4-hydroxycinnamic acid, 4HCA, Figure 1b) created high-performance, biodegradable, plant-derived plastic resins with high heat resistance over $150^{\circ} \mathrm{C} .{ }^{13}$ Because DHCA tends to introduce branching points, the copolymer resin is hyper-branched and has many catechol groups at its chainends as shown in Figure 1c. This resin can be expected to show adhesive characteristics to various kinds of surfaces, ${ }^{14}$ such as those of the Mussel proteins. ${ }^{15}$ In this paper, we report the strong adhesive characteristics of Mussel-mimetic bio-based resin to organic and inorganic surfaces.

\section{EXPERIMENTAL PROCEDURE}

Synthesis of poly(DHCA-co-4HCA)

Biomass monomers of caffeic acid (4HCA, DHCA; Tateyama Kasei, Co. Ltd., Imizu city, Japan) and p-coumaric acid (4HCA; Tateyama Kasei, Co. Ltd.) were used as received. The chemical structures of the biomass monomers are shown in Figure 2. It shows caffeic acid with a catechol group, and Figure $2 \mathrm{~b}$ shows p-coumaric acid with a rigid moiety. Copolymer resin of poly (DHCA-co-4HCA) was synthesized by the polymerization of $4 \mathrm{HCA}$ and DHCA with a sodium acid phosphate catalyst under flowing $\mathrm{N}_{2}$ gas for $3 \mathrm{~h}$ at $200^{\circ} \mathrm{C}$ in a separable flask. Afterward, vacuum was drawn on the separable flask to a pressure of reactant molecules for $6 \mathrm{~h}$ in order to remove the acetic acid generated during the polymerization. The details of these procedures have been described elsewhere. ${ }^{13}$

\section{Melting adhesive bonding}

Carbon and glass substrates were obtained from the Nilaco Corporation (Tokyo, Japan) and Sigma Koki Co., Ltd. (Tokyo, Japan), respectively, and used as received. Both the carbon and glass substrates were $50 \mathrm{~mm}$ long, $50 \mathrm{~mm}$ wide and $5 \mathrm{~mm}$ thick. Steel substrates were obtained from Three Bond Co. Ltd. (Hachiohji, Japan), measuring $100 \mathrm{~mm}$ long, $25 \mathrm{~mm}$ wide and $0.8 \mathrm{~mm}$ thick. The adhesive poly(DHCA-co-4HCA) resin was melted between substrates at $200^{\circ} \mathrm{C}$ and $5 \mathrm{MPa}$ for $5 \mathrm{~min}$ using a hot press. The bonding area was $10 \mathrm{~mm}$ long and $50 \mathrm{~mm}$ wide in the case of the carbon and glass substrates and $10 \mathrm{~mm}$ long and $25 \mathrm{~mm}$ wide in the case of the steel substrates. The bonding samples were then cooled to room temperature under atmospheric conditions.

Tensile test for shear adhesion

The shear adhesion test was carried out with a tensile testing machine (3365-L5, Instron, Canton, MA, USA). As shown in the upper illustration of Figure 1, the bonding samples were held by two fixtures near the midpoint of the sample

${ }^{1}$ School of Materials Science, Japan Advanced Institute of Science and Technology, Nomi city, Ishikawa, Japan and ${ }^{2}$ School of Knowledge Science, Japan Advanced Institute of Science and Technology, Nomi city, Ishikawa, Japan

Correspondence: Professor T Kaneko, School of Materials Science, Japan Advanced Institute of Science and Technology, Nomi city, Ishikawa 923-1292, Japan.

E-mail: kaneko@jaist.ac.jp

Received 16 February 2011; revised 19 June 2011; accepted 27 June 2011; published online 17 August 2011 

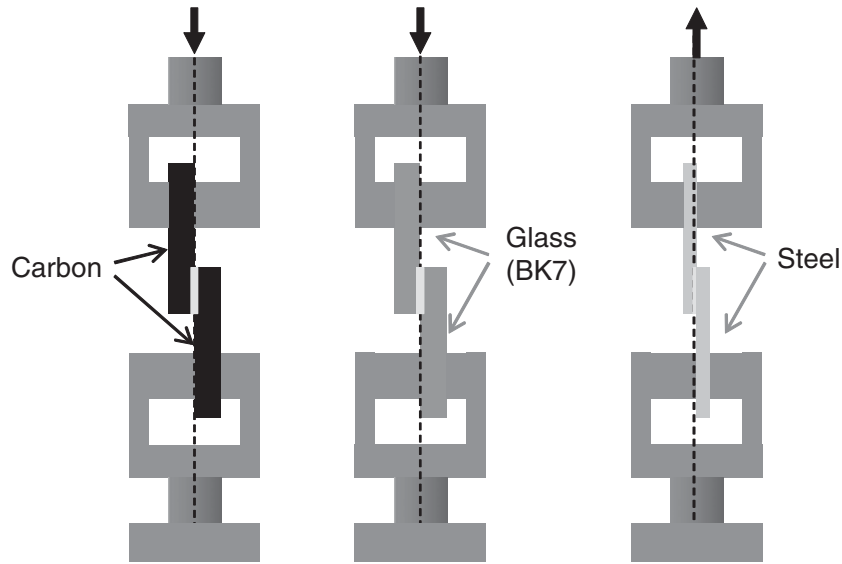

Figure 1 Schematic illustration of the adhesion test. Pairs of carbon, glass and steel substrates are glued by poly (DHCA-Co-4HCA) at $200^{\circ} \mathrm{C}$ for $10 \mathrm{~min}$. The adhesion area of carbon and glass is $1 \times 5 \mathrm{~cm}$; that of steel is $1 \times 2.5 \mathrm{~cm}$. A full color version of this figure is available at Polymer Journal online.<smiles>O=C(O)/C=C/c1ccc(O)c(O)c1</smiles>

b<smiles>O=C(O)/C=C/c1ccc(O)cc1</smiles>

C

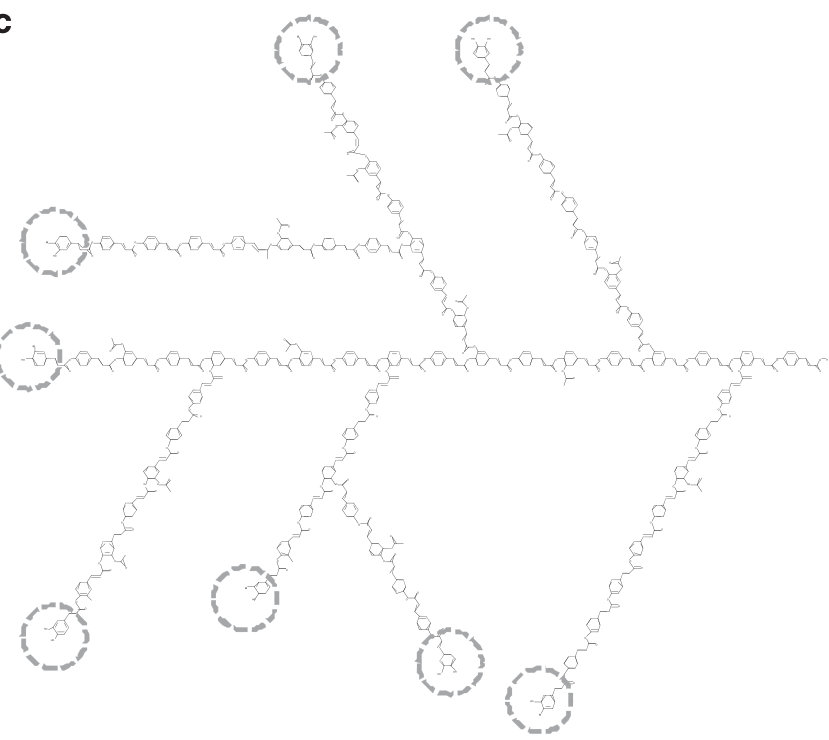

Figure 2 Chemical structure of (a) 3,4-dihydroxycinnamic acid (DHCA) and (b) 4-hydroxycinnamic acid (4HCA). (c) Chemical structure of poly(DHCA-co$4 \mathrm{HCA}$ ) resin determined by proton nuclear magnetic resonance, infrared and gel-permeation chromatography. Polymer chains were hyper-branched, and catechol groups exist at their chain ends (dotted circles). A full color version of this figure is available at Polymer Journal online.

and pulled in opposite directions. The force and the displacement of the fixture were simultaneously monitored, and the stress was defined as the force divided by the bonding area. For the comparison measurement, conventional instant superglue (Aron Alpha, Toagosei Co., Ltd., Tokyo, Japan) was used.

\section{RESULTS AND DISCUSSION}

\section{Synthesis of poly(DHCA-co-4HCA) resin}

From an analysis of the infrared, gel-permeation chromatography and proton nuclear magnetic resonance spectra, the chemical structure of
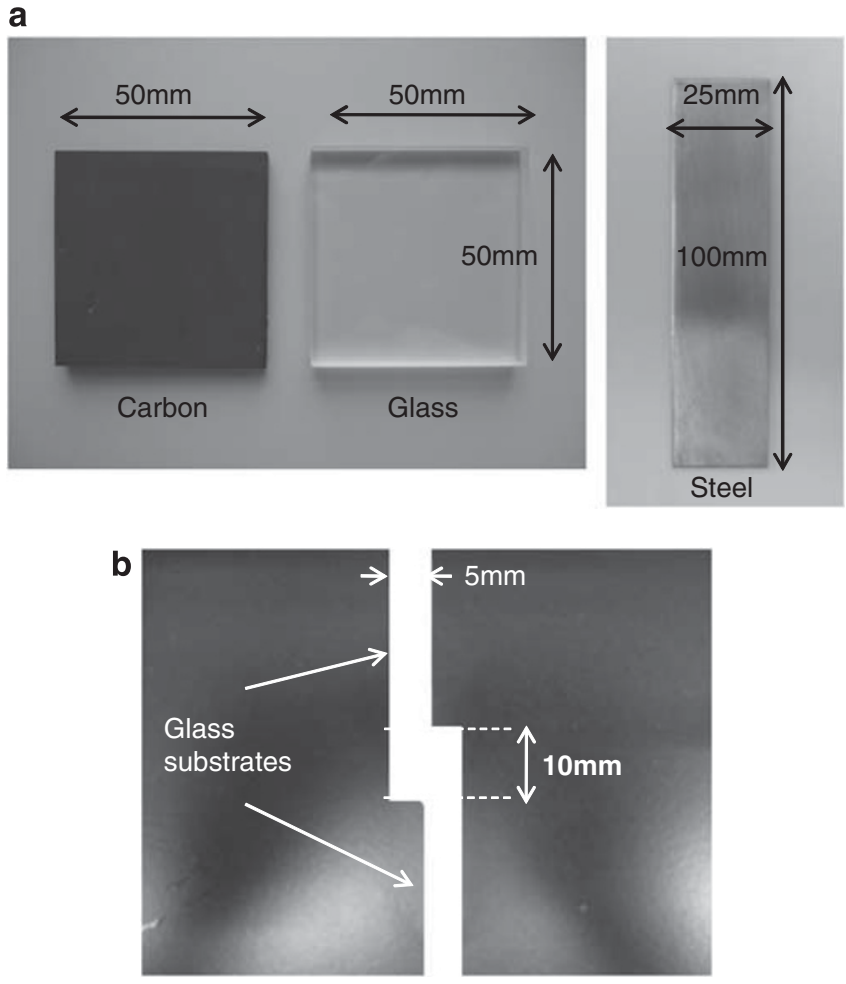

Figure 3 (a) Surface features of the carbon, glass and steel substrates. The carbon and glass substrates are $5 \mathrm{~mm}$ in thickness, $50 \mathrm{~mm}$ in length and $50 \mathrm{~mm}$ in width. The steel substrates are $0.8 \mathrm{~mm}$ in thickness, $100 \mathrm{~mm}$ in length and $50 \mathrm{~mm}$ in width. (b) Cross-section of a glass substrate glued by poly(DHCA-co-4HCA) resin. The glued area is $10 \mathrm{~mm}$ in length $\times 50 \mathrm{~mm}$ in width. A full color version of this figure is available at Polymer Journal online.

Table 1 Evaluation of the surface roughness of carbon, glass and steel by atomic force mirsoscopy

\begin{tabular}{lccc}
\hline & Carbon & Glass & Steel \\
\hline RMS $(\mathrm{nm})$ & 1154 & 1.9 & 4744 \\
\hline
\end{tabular}

Abbreviation: RMS, root mean square.

poly(DHCA-co-4HCA) resin was determined as shown in Figure 2c. Because the copolymers have a rigid main chain composed primarily of $p$-substituted benzene rings, the main chain should be rigid and show liquid crystalline properties. Another characteristic of the copolymer is the hyper-branched structure with a high density of catechol groups at the chain ends. This may produce strong interactions with the surfaces of various materials. ${ }^{1-3,5}$

\section{Sample preparation of carbon and glass substrates glued by} poly(DHCA-co-4HCA) resin

As mentioned above, the catechol group can adhere strongly to organic or inorganic surfaces. We have prepared the carbon substrate as a representation of organic surfaces, and the glass and steel substrates as representations of inorganic surfaces. As shown in Figure 3a, carbon and glass substrates $(50 \times 50 \times 5 \mathrm{~mm})$ and steel $(100 \times 25 \times 0.8 \mathrm{~mm})$ substrates were used. The surface roughness of each substrate was evaluated by tapping mode atomic force microscopy (Veeco, Nano Scope IV, Plainview, NY, USA), the results of which are summarized in Table 1. 

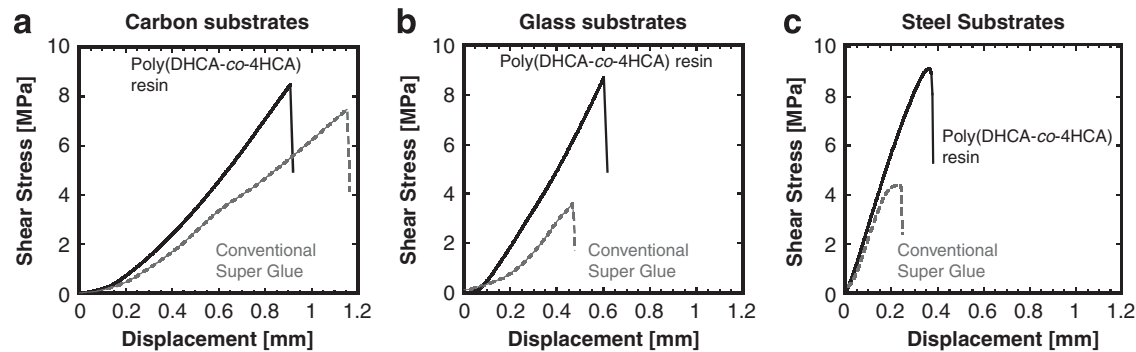

Figure 4 Typical stress-displacement curve of the shear adhesion tests between (a) carbon substrates, (b) glass substrates and (c) steel substrates. The solid line in the graph denotes the substrates glued by poly(DHCA-co-4HCA) resin, and the dotted line denotes the substrates glued by conventional instant superglue. A full color version of this figure is available at Polymer Journal online.

\section{Tensile test experiment}

As shown in Figure 1, the non-glued areas of the carbon and glass substrates were held by a fixture of the tensile tester apparatus and pulled in opposite directions to measure the adhesive shear stress. The raw data for the carbon substrates, glass substrates and steel substrates are shown in Figures $4 \mathrm{a}, \mathrm{b}$ and $\mathrm{c}$, respectively. For comparison, conventional instant superglue (Aron Alpha, Toagosei Co., Ltd.) was also evaluated by the same methods. The stress-displacement curves shown by solid lines in Figures $4 \mathrm{a}, \mathrm{b}$ and $\mathrm{c}$ are for substrates glued by poly(DHCA-co-4HCA) resin, and the stress curves shown by dotted lines in the same figure are for substrates glued by conventional instant superglue. In Figure 4a, the maximum fracture stress of poly(DHCA-co4HCA) was over $9 \mathrm{MPa}$ and somewhat larger than that of conventional instant superglue. This result indicates that poly(DHCA-co-4HCA) resin can effectively adhere to the organic substrate. This strong adhesive characteristic may result from the formation of strong hydrogen bonds because the carbon surface was exposed to air and instantly oxidized. On the other hand, the fracture stress of the conventional instant superglue was $\sim 7 \mathrm{MPa}$, which may result from a strong anchor effect due to penetration into the carbon substrates. This value was within the expected performance of conventional instant superglue $(2 \sim 8 \mathrm{MPa})$. In Figure $4 \mathrm{~b}$, poly(DHCA-co-4HCA) resin and conventional instant superglue are evaluated for their ability to adhere to very flat glass surfaces (BK7). The fracture stress of poly(DHCA-co-4HCA) resin between glass substrates was also over $9 \mathrm{MPa}$. This strong adhesive characteristic might also result from strong hydrogen bonding because the glass surface usually has $\mathrm{OH}$ groups on it. On the other hand, conventional instant superglue cannot exceed $4 \mathrm{MPa}$ on these surfaces because it cannot show anchor effects. In the case of the steel substrates, the adhesion results for poly(DHCA-co-4HCA) resin were similar to those for glass substrates. The adhesive force of conventional instant superglue remained lower ( $\sim 4 \mathrm{MPa})$ than its value for the carbon substrate, despite the greater roughness of the steel surface (Table 1). This result may indicate that conventional instant superglue cannot penetrate into steel.

Figure 5 is a summary of the adhesion tests. From Figure 5, it is clear that the adhesive characteristics of poly(DHCA-co-4HCA) resin are superior to those of conventional instant superglue. The adhesive properties of conventional instant superglue are inferior when applied to the flat surface, which cannot be expected to show anchor effects. On the other hand, poly(DHCA-co-4HCA) resin demonstrated its adhesive properties even on the very flat glass surfaces. This may result from the hydrogen bond effect between the $\mathrm{OH}$ groups of catechol and the glass surfaces.

As discussed above, most of the substrate surfaces were oxidized and have the potential to make strong hydrogen bonds with the

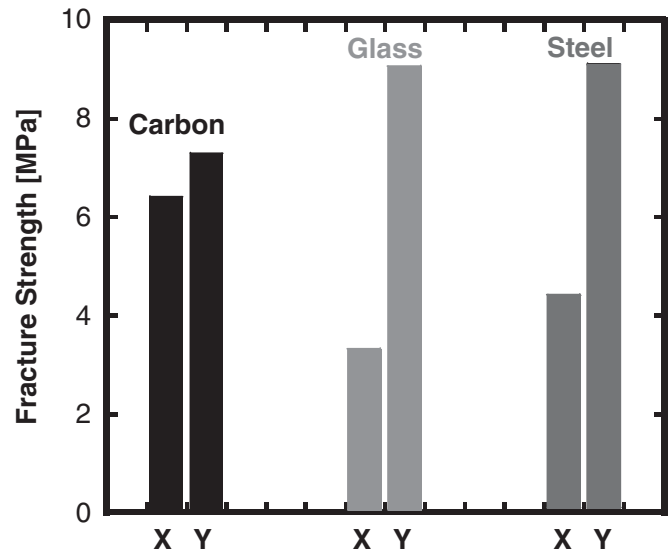

Figure 5 Fracture strength of glued substrates (carbon, glass, steel). $X$ means that each substrate was glued by conventional instant superglue, and $Y$ means that they were glued by the poly(DHCA-co-4HCA) resin. A full color version of this figure is available at Polymer Journal online.

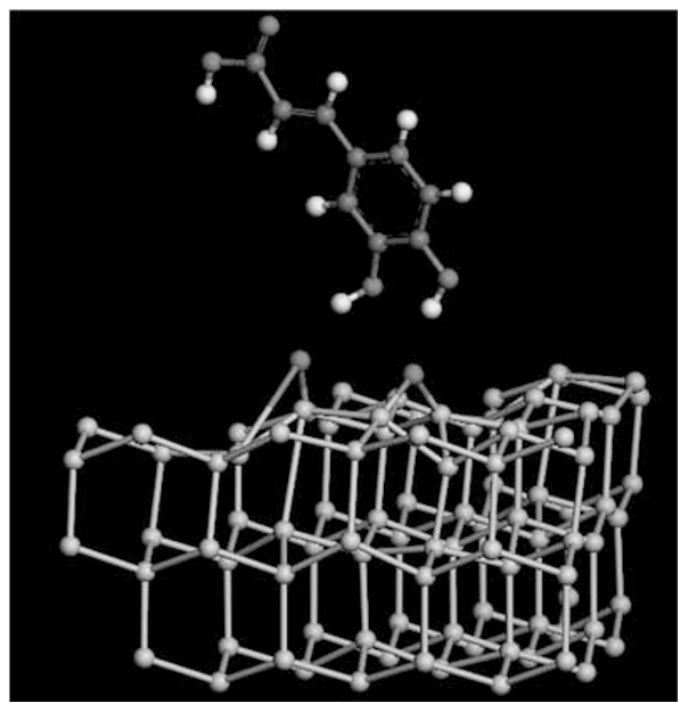

Figure 6 Simulation results of the interaction between caffeic acid and partially oxidized silicon crystal using quantum chemical calculation (Dmol3). A full color version of this figure is available at Polymer Journal online. 
catechol groups at the chain ends of our adhesive resin, which has electron-releasing substituents such as hydroquinone.

Figure 6 shows an example of simulation results obtained by quantum chemical calculation (Dmol3) using a model of caffeic acid and partially oxidized silicon as an adhesive interface. The figure illustrates an adhesive state $(3 \times 3)$ between caffeic acid and the partially oxidized $\mathrm{Si}(1 \times 1 \times 1)$ surface. Non-oxidized surfaces of silicon are terminated by hydrogen, which was omitted here. It is worth noting that a stable hydrogen bond was formed between the catechol group and oxygen on the substrate. Owing to this interaction, silicon atoms between two oxygens were displaced primarily at the partially oxidized surface, with the strain seeming to affect only the silicon surface. The distance between oxygen and oxygen is around $2 \AA$, which is much smaller than that normally seen for water $(2.7 \AA)$. This result indicates that reassembly at the interface is a contributing factor to adhesion in addition to singular hydrogen bonds. In other words, adhesion is an integrated phenomenon between the adhesive polymer resin and substrates; therefore, we must understand the interaction stability of the surface states of both the adhesive polymer resin and substrates.

\section{SUMMARY}

We have synthesized a novel adhesive resin of poly(DHCA-co4HCA) from biomass monomers that has catechol groups at the chain ends. Because the catechol groups interact strongly with organic/inorganic surfaces, we have confirmed the adhesive properties using our novel adhesive resin. Because our novel adhesive resin was made from biomass monomers and has a benzene ring in the main chain, it is expected to be environmentally friendly and non-toxic.

We are now working to produce novel resins with stronger adhesion than poly(DHCA-co-4HCA), which is comparable to epoxy resin.

\section{ACKNOWLEDGEMENTS}

This research was supported by the Sasakawa Scientific Research Grant from the Japan Science Society, a Grant-in-Aid for JST (Practical application research, Kaneko Project) and the Iketani Science and Technology Foundation. We sincerely thank Professor Tadaoki Mitani for his valuable discussions.

1 Herbart Waite, J. \& Tanzer, M. L. Polyohenolic substance of Mytilus edulis: novel adhesive containing L-dopa and hydroxyproline. Science 212, 1038-1940 (1981).

2 Lin, Q., Gourdon, D., Sun, C., Holten-Andersen, N., Anderson, T. H., Waite, J. H. \& Israelachvili, J. N. Adhesion mechanisms of the mussel foot proteins mfp-1 and mfp-3'. Proc. Natl Acad. Sci. USA 104, 3782-3786 (2007).

3 Weigemann, M. Adhesion in blue mussels (Mytilus edulis) and barnacles (genus Balanus): mechanisms and technical allocation. Aquat. Sci. 67, 166-176 (2005).

$4 \mathrm{Yu}$, M. \& Deming, T. J. Synthetic polypeptide mimics of marine adhesives. Macromolecules 31, 4739-4745 (1988).

5 Yu, M., Hang, J. \& Deming, T. J. Role of L-3,4-dihydroxyphenylalanine in mussel adhesive proteins. J. Am. Chem. Soc. 121, 5825-5826 (1999).

6 Lee, H., Scerer, N. F. \& Messersmith, P. B. Single-molecule mechanics of mussel adhesion. Proc. Natl Acad. Sci. USA 103, 12999-13003 (2006).

7 Waite, J. H. Mussel power. Nat. Mater. 7, 8-9 (2008).

8 Lee, H., Lee, B. P. \& Messersmith, P. B. A reversible wet/dry adhesive inspired by mussels and geckos. Nature 448, 338-341 (2007).

9 Gunawan, R. C., King, J. A., Lee, B. P., Messersmith, P. B. \& Miller, W. M. Surface presentation of bioactive ligands in a nonadhesive background using DOPA-tethered biotinylated poly(ethylene glycol). Langmuir 23, 10635-10643 (2007).

10 Lee, H., Dellatore, S. M., Miller, W. M. \& Messersmith, P. B. Mussel-inspired surface chemistry for multifunctional coatings. Science 318, 426-430 (2007).

11 Lee, H., Lee, Y., Statz, A. R., Rho, J., Gwan Park, T. \& Messersmith, P. B. Substrateindependent layer-by-layer assembly by using mussel-adhesive-inspired polymers. Adv. Mater. 20, 1619-1623 (2008).

12 Lee, H., Rho, J. \& Messersmith, P. B. Facile conjugation of biomolecules onto surfaces via mussel adhesive protein inspired coatings. Adv. Mater. 21, 431-434 (2009).

13 Kaneko, T., Thi, T. H., Shi, D. J. \& Akashi, M. Environmentally degradable high-performance thermoplastics from phenolic phytomonomers. Nat. Mater. 5, 966-970 (2006).

14 Pizzi, A. Tannin Adhesives, 'Advanced Wood Adhesives Technology, (Marcel Dekker Inc. New York, 1994)

15 Kaneko, D., Kinugawa, S., Matsumoto, K. \& Kaneko, T. Terminally-catecholized hyperbranched polymers with high performance adhesive characteristics. Plant Biotech. 27, 293-296 (2010). 